\title{
IL SOGGIORNO DI SANT'ILARIONE DI GAZA IN DALMAZIA SECONDO LA VITA S. HILARIONIS DI SAN GIROLAMO
}

L'articolo intende presentare brevemente il soggiorno di sant'Ilarione di Gaza ad Epidauro in Dalmazia, e ciò secondo la Vita S. Hilarionis, stilata da san Girolamo all'inizio della permanenza betlemita dell'Autore, dopo i viaggi in Siria e in Egitto, cioè dopo gli anni 386-390, ma non oltre il $396^{1}$.

Il protagonista dell'opera è Ilarione, monaco vissuto tra il 291 e il 371, fondatore del monachesimo in Palestina, nativo di Tabatha, vicino a Gaza, città pagana dedita al culto di Marnas e convertita al cristianesimo intorno al V sec., grazie alla predicazione di san Porfirio. Oltre a queste notizie, non si sa da quali fonti san Girolamo abbia tratto gli elementi della sua Vita perché, nonostante la celebrità, di Ilarione non si scrisse nulla prima della Vita; sappiamo soltanto dallo stesso san Girolamo, nell'introduzione ${ }^{2}$, che sant'Epifanio di Salamina aveva scritto un suo elogio, andato purtroppo perduto.

Sant'Ilarione, per evitare il contatto con la gente e mantenere la sua ascesi eremitica, cercò di continuo di abitare in un posto appartato e deserto, aiutato in ciò dal fedele discepolo Esichio che lo condusse proprio ad Epidauro in Dalmazia. Presentando brevemente questo suo soggiorno, san Girolamo descrive due miracoli compiuti dal Santo: la liberazione della popolazione dall'azione devastante di un enorme e feroce drago, e il calmare lo tsunami che minacciava la popolazione con una vera e propria distruzione. In tal modo, presentiamo questi due miracoli, avvenuti in Dalmazia, dividendo il nostro articolo in due parti.

1. Sant'Ilarione brucia un drago. Durante una delle sue fughe e dei suoi spostamenti in cerca delle condizioni propizie per poter contemplare Dio in solitudine, sant'Ilarione giunse ad Epidauro, in Dalmazia. Ecco i testi che parlano di questo suo spostamento, ma anche del primo miracolo da lui compiuto in questa terra lontana.

* Prof. dr. hab. Bazyli Degórski, O.S.P.P.E. - professore di patrologia e di teologia dogmatica dell'età patristica presso la Pontificia Università S. Tommaso d'Aquino a Roma, e professore di patrologia e di antropologia patristica alla Pontificia Facoltà Teologica "Teresianum" a Roma; procuratore generale presso la Santa Sede; e-mail: osppe.roma@gmail.com.

${ }^{1}$ Cf. J. Gribomont Ilarione di Gaza, DPAC II 1753 (anche in: NDPAC II 2528).

${ }^{2} \mathrm{Cf}$. Hieronymus, Vita S. Hilarionis 1, 5. 
"E per non attardarmi oltre, il sant'uomo Esichio si gettò alle ginocchia del maestro e «gli rigò i piedi di lacrime»e, infine, da lui fu rialzato. Dopo due o tre giorni di conversazione, udì da Zanano che il vecchio non poteva più abitare in quelle regioni, ma voleva proseguire verso alcune popolazioni barbare, dove sia il suo nome, che la sua lingua fossero ignoti"3.

"Esichio lo condusse, dunque, a Epidauro, città della Dalmazia, dove si fermò per pochi giorni in un vicino campicello, senza, però, poter rimanere nascosto. Giacché un drago di fenomenale grandezza - di quelli che nella lingua del posto vengono chiamati «boa», poiché sono tanto grossi che sogliono ingoiare buoi - devastava tutta la provincia in lungo e in largo e inghiottiva non solo armenti e greggi, ma anche contadini e pastori, attirati a sé con la forza del suo fiato, ordinò di preparare per lui un rogo e, avendo rivolto una preghiera a Cristo, fece venire il drago e, dopo avergli ordinato di salire sulla catasta di legna, appicò il fuoco. In questo modo, di fronte a tutto il popolo, bruciò quella bestia immane"4.

"Epidauro": si tratta di Ragusa Vecchia, l'odierna Dubrovnik". La fisionomia di per sé repellente di animali come questo, alimentava ancor più nelle popolazioni di alcune regioni la paura verso rettili di maggiori proporzioni, sui cui dati oggettivi s'innestava allora il racconto popolare che ne ingigantiva 1'immagine terrorizzante, proiettandovi gli spettri della propria impressionabilità 6 .

${ }^{3}$ Ibidem 28, 1, ed. B. Degórski, in: Hieronymi, Historica et hagiographica. Vita Beati Pauli monachi Thebaei. Vita Hilarionis. Vita Malchi monachi captivi. Epistula praefatoria in Chronicis Eusebii Caesariensis. Chronicorum Eusebii Caesariensis continuatio. De viris inlustribus. In Regulae S. Pachomii versionem praefatio = Girolamo, Opere storiche e agiografiche. Vita di san Paolo, eremita di Tebe. Vita di Ilarione. Vita di Malco, l'eremita prigioniero. Prefazione alla traduzione delle Cronache di Eusebio di Cesarea. Continuazione delle Cronache di Eusebio di Cesarea. Gli uomini illustri. Prefazione alla traduzione della Regola di Pacomio, Hieronymi Opera $15=$ Opere di Girolamo 15, Roma 2014, 168-171: "Et ne longum faciam, sanctus vir Hesychius ad magistri genua provolutus «plantasque eius lacrimis rigans» $(\operatorname{Lc} 7,38)$ tandem ab eo sublevatus, post bidui triduique sermonem audit a Zanano, non posse senem iam in illis habitare regionibus, sed velle ad barbaras quasdam pergere nationes, ubi et nomen et sermo suus incognitus foret. Duxit itaque illum ad Epidaurum, Dalmatiae oppidum, ubi paucis diebus in vicino agello mansitans non potuit abscondi".

${ }^{4}$ Ibidem 28, 2-3, Hieronymi Opera 15, 170-171: "Duxit itaque illum ad Epidaurum, Dalmatiae oppidum, ubi paucis diebus in vicino agello mansitans non potuit abscondi. Siquidem draco mirae magnitudinis, quos gentili sermone «boas» vocant, eo quod tam grandes sint, ut boves glutire soleant, omnem late vastabat provinciam, et non solum armenta et pecudes, sed agricolas quoque et pastores, tractos ad se vi spiritus, absorbebat. Cui cum pyram iussisset praeparari et oratione ad Christum emissa evocato praecepisset struem lignorum scandere, ignem supposuit. Cunctaque spectante plebe immanem bestiam concremavit".

${ }^{5}$ Cf. G. Praga, La leggenda di S. Ilarione a Epidauro, "Archivio Storico della Dalmazia" 25 (1938) 81-91; I. Opelt, Des Hieronymus Heiligenbiographien als Quellen der historischen Topographie des östlichen Mittelmeerraumes, RQ 74 (1979) 172.

${ }^{6}$ Cf. B. Degórski, La natura del deserto nelle "Vitae" di san Girolamo, in: La cultura scientifico-naturalistica nei Padri della Chiesa (I-V sec.). XXXV Incontro di studiosi dell'antichità cristiana, Roma, 4-6 maggio 2006, Istituto Patristico “Augustinianum”, SEA 101, Roma 2007, 573-574. 
Per quanto riguarda l'espressione geronimiana "nella lingua del paese" (gentili sermone), bisogna dire che si tratta del dialetto parlato dalle gente semplice in quel luogo citato della Dalmazia, anche se la lingua latina conosce il sostantivo boas (boae) che è un sostantivo maschile della I declinazione ${ }^{7}$, e questa forma viene proprio qui adoperata da san Girolamo. Bisogna dire che nella lingua latina troviamo anche il sostantivo bŏă (boae) che è un sostantivo femminile della I declinazione ${ }^{8}$. Ciò nonostante, san Girolamo, esimio cultore della bellezza dello stile latino, considera questa parola - boas - barbara ${ }^{9}$.

Va inoltre ricordato che l'antichità conosceva questo tipo di serpente. Così, ad esempio, attesta in proposito pure Plinio il Vecchio nell'VIII libro della sua monumentale opera dedicata proprio alla zoologia degli animali terrestri:

"Megastene scrive che in India i serpenti crescono fino ad una tale grandezza, che inghiottono interi cervi e tori. Metrodoro scrive che nei dintorni del fiume Rindaco nel Ponto inghiottono, risucchiati con il fiato, uccelli che volano, anche se in alto e rapidamente. È conosciuto un serpente, lungo centoventi piedi, attaccato come una fortezza con balliste e catapulte dal comandante Regolo durante le guerre puniche presso il fiume Bagrada. La sua pelle e le sue mascelle si conservarono in un tempio a Roma fino alla guerra contro Numanzia. A queste cose dànno credenza quelli chiamati in Italia «bova», che raggiungono tanta grandezza che sotto l'imperatore Divino Claudio si è visto un [intero] bambino nel ventre di uno [di questi] ucciso sul Vaticano. Al primo succhiare vengono nutriti di latte di vacca, da qui presero il nome" ${ }^{\prime 10}$.

Il racconto geronimiano, riguardante gli spostamenti e le vicissitudini di sant'Ilarione in Dalmazia, sempre in cerca dell'amato Dio, continua:

${ }^{7}$ Cf., ad esempio, Dizionario latino, in: http://www.dizionario-latino.com/dizionario-latinoflessione.php?lemma=BOAS100 (consultato il 9 I 2017).

${ }^{8}$ Cf., ad esempio, Dizionario latino, in: http://www.dizionario-latino.com/dizionario-latinoitaliano.php?lemma=BOA100 (consultato il 9 I 2017).

${ }^{9}$ San Girolamo adopera l'espressione gentili sermone ("nella lingua del paese") anche nella Vita S. Pauli Primi Eremitae $(6,2)$ parlando, questa volta, della lingua siriaca. Il Dalmata usa tale espressione anche se il siriaco era la lingua semitica più importante dell'Oriente cristiano. Esso era molto vicino al dialetto usato da Gesù Cristo. Infatti, il siriaco viene definito come dialetto aramaico tardivo, derivante dal ramo comune semitico nord-occidentale. Questa lingua divenne lingua letteraria proprio nel sec. IV, quando Girolamo stilò la sua opera (375-377) e la maggior parte della popolazione si convertì al cristianesimo.

${ }^{10}$ Gaius Plinius Secundus, Naturalis historia VIII 14, 36-37, in: http://penelope.uchicago.edu/ Thayer/L/Roman/Texts/Pliny_the_Elder/8*.html (consultato il 9 I 2017): "Megasthenes scribit in India serpentes in tantam magnitudinem adolescere, ut solidos hauriant cervos taurosque, Metrodorus circa Rhyndacum amnem in Ponto, supervolantes ut quamvis alte perniciterque alites haustu raptas absorbeant. Nota est in Punicis bellis ad flumen Bagradam a Regulo imperatore ballistis tormentisque, ut oppidum aliquod, expugnata serpens CXX pedum longitudinis; pellis eius maxillaeque usque ad vellum Numantinum duravere Romae in templo. faciunt his fidem in Italia appellatae bovae in tantam amplitudinem exeuntes, ut Divo Claudio principe occisae in Vaticano solidus in alvo spectatus sit infans. aluntur primo bubuli lactis suctu, unde nomen traxere", trad. italiana di B. Degórski. 
"In seguito, si preparava a un'altra fuga, deliberando molto sollecitamente che cosa fare e verso dove dirigersi e, percorrendo con la mente le terre solitarie, si addolorava che, anche se la lingua taceva su di lui, i miracoli parlassero""11.

Sant'Ilarione delibera accuratamente il piano della sua fuga davvero rocambolesca. Per esprimerlo, san Girolamo si serve, tra l'altro, dell'espressione "verso dove dirigersi" (quo se verteret). Si tratta di un'espressione caratteristica della tradizione classica, che viene adoperata, ad esempio, da Publio Terenzio Afro nella sua commedia "Hecyra" (o "La suocera"), ove Mirrina, moglie di Fidippo, madre di Filumena, si chiede tutta costernata come il nostro sant' Ilarione: "Che cosa fare? Sono perduta. Dove posso rivolgermi? Povera me!"12, oppure da Marco Tullio Cicerone nelle "Filippiche" che egli pronunciò contro Marco Antonio dal 2 settembre del 44 a.C. al 21 aprile del 43 a.C. (eccezione fatta proprio per la II "Filippica", immaginata come pronunciata in senato, in risposta agli attacchi di Marco Antonio nei suoi riguardi durante la riunione del 19 settembre, alla quale lo stesso Cicerone non prese parte):

"Questo prodigio nondimeno proibirono gli eredi di L. Rubrio per decreto di

Cesare. Rimaneva confuso il fellone, non avendo dove rivolgersi"13.

San Girolamo adopera quest'espressione anche altrove ${ }^{14}$.

2. A Epidauro, sant'Ilarione doma il mare. Segue la descrizione di un altro miracolo compiuto da sant'Ilarione ad Epidauro, in Dalmazia: il nostro monaco e taumaturgo, preso dalla compassione per la povera e disgraziata popolazione vessata dalle forze della natura o, meglio, dallo stesso Dio, doma

${ }^{11}$ Hieronymus, Vita S. Hilarionis 28, 2-5, Hieronymi Opera 15, 170-171: "Duxit itaque illum ad Epidaurum, Dalmatiae oppidum, ubi paucis diebus in vicino agello mansitans non potuit abscondi. 3. Siquidem draco mirae magnitudinis, quos gentili sermone «boas» vocant, eo quod tam grandes sint, ut boves glutire soleant, omnem late vastabat provinciam, et non solum armenta et pecudes, sed agricolas quoque et pastores, tractos ad se vi spiritus, absorbebat. 4. Cui cum pyram iussisset praeparari et oratione ad Christum emissa evocato praecepisset struem lignorum scandere, ignem supposuit. Cunctaque spectante plebe immanem bestiam concremavit. 5. Unde aestuans quid faceret, quo se verteret, aliam parabat fugam et solitarias terras mente perlustrans maerebat, quod tacente de se lingua miracula loquerentur".

${ }^{12}$ Publius Terentius Afer, Hecyra 4, 1, 516-517, in: http://atin.packhum.org/loc/134/5/4\#4 (consultato il 9 I 2017): "Perii, quid agam? Quo me vortam? Quid viro $\mathrm{m}<\mathrm{eo}>$ respondebo misera?", trad. italiana di B. Degórski.

${ }^{13}$ Marcus Tullius Cicero, Philippicae 2, 29, 74, in: https://la.wikisource.org/wiki/In_M._Antonium_Oratio_-_Philippica_II\#.5B29.5D (consultato il 9 I 2017): "Hanc tamen auctionem heredes L. Rubri decreto Caesaris prohibuerunt. Haerebat nebulo; quo se verteret, non habebat", trad. italiana di B. Degórski.

${ }^{14}$ Cf., per es., Hieronymus, Vita S. Pauli Primi Eremitae 9, 1, ed. B. Degórski, in: Hieronymi Opera 15, 98-99: "Quid ageret, quo verteret gradum?" ("Che cosa fare? Dove dirigere i suoi passi?”); idem, Vita S. Hilarionis 3, 2, Hieronymi Opera 15, 122-123: “Quid faceret diabolus? Quo se verteret? (Che cosa avrebbe potuto fare il diavolo? A che cosa si sarebbe volto?”). 
il mare, compiendo in tal modo, con la forza del Signore, che opera nel suo eletto, un altro grandissimo ed evidente miracolo:

"In quel tempo, a causa di un terremoto, avvenuto in tutto il mondo dopo la morte di Giuliano, i mari uscirono dai loro confini e, quasi che Dio di nuovo minacciasse il diluvio ${ }^{15}$ o tutto tornasse nell'antico caos, le navi, sollevate alle vette dei monti, restarono lì appese in aria"16.

Questo accadde durante l'impero di Giuliano l'Apostata, detto anche "il Trasgressore" (361-363), il quale tentò di restaurare il paganesimo ellenistico, ricorrendo a vere e proprie persecuzioni contro i cristiani. Per esempio, a Gaza, città tradizionalmente pagana, devota all'idolo Marnas, si scatenò una crudele persecuzione popolare contro i cristiani. Di ciò narra lo storico cristiano Sozomeno $(\uparrow 450 \text { circa })^{17}$. Questo fatto, riguardante il terremoto, che causò la detta inondazione, avvenne esattamente il 21 luglio del 365 (o del 366). Ciò viene anche raccontato dallo storico pagano Ammiano Marcellino ${ }^{18}$ e da tre storici cristiani: Orosio ${ }^{19}$, Socrate $^{20}$ e Sozomeno ${ }^{21}$. Lo stesso san Girolamo menziona questa specie di tsunami, con un'espressione simile a quella adoperata qui, anche due volte altrove: una volta nel Commento al Profeta Isaia, e l'altra nella Continuazione delle "Cronache" di Eusebio di Cesarea:

"Da un abitante di Areopoli ho sentito dire (ma la città intera lo può testimoniare) che, quando per un grave terremoto, avvenuto al tempo della mia infanzia, ovunque il mare oltrepassò le spiagge, in quella stessa notte crollarono le mura della città $[=\text { della città di Ar, di Areopoli }]^{\prime 22}$.

${ }^{15} \mathrm{Cf}$. Gn 6, 17. A proposito dell'interpretazione patristica del diluvio, cf. A. Wilmart, Arca Noë, RBen 26 (1909) 1-12; J. Daniélou, Déluge, baptême, jugement, "Dieu vivant" 8 (1947) 97-112; idem, Sacramentum futuri. Études sur les origines de la typologie biblique, Paris 1950, 55-94 (Noé et le déluge); P. Féghali, La figura di Noè secondo i SS. Padri (Contributo alla storia della esegesi), "Studii biblici Franciscani liber annuus" 20 (1970) 138-165; H. Boblitz, Die Allegorese der Arche Noah in der frühen Bibelauslegung, "Frühmittelalterliche Studien" 6 (1972) 159-170; P. Féghali, Note sur l'exégèse de Saint Éphrem. Commentaire sur le déluge (Gn 6, 19-9. 17), ParOr 8 (19771978) 67-86.

${ }^{16}$ Per quanto riguarda questo brano, cf. Degórski, La natura del deserto nelle Vitae di san Girolamo, p. 549-578; idem, Przyroda pustyni jako „,stworzenie” u św. Hieronima, in: Wiedza w stużbie dobra człowieka, ed. M. Włosiński, Włocławek 2010, 71-82.

${ }^{17}$ Cf. Sozomenus, HE V 3, 6-8; 9, 1-3. Cf. anche Hieronymus, Vita S. Hilarionis 23, 6.

${ }^{18} \mathrm{Cf}$. Ammianus Marcellinus, Res gestae XXVI 10, 15-19.

${ }^{19} \mathrm{Cf}$. Paulus Orosius, Historia adversus paganos VII 32, 5.

${ }^{20} \mathrm{Cf}$. Socrates, HE IV 3.

${ }^{21}$ Cf. Sozomenus, HE V 10, 2.

${ }^{22}$ Hieronymus, Explanationes in Esaiam V 44 (15, 1-2b), ed. R. Maisano: Hieronymi, Explanationes in Esaiam. Girolamo. Commento a Isaia, Hieronymi Opera 4/2, Roma 2013, 48-49: "Audivi quendam Areopoliten, sed et omnis civitatis testis est, motu terrae magno in mea infantia, quando totius orbis litus transgressa sunt maria, eadem nocte muros urbis istius corruisse". 
"In seguito ad un terremoto, fattosi sentire per tutta la terra, la costa è invasa dal mare e in molte città della Sicilia e nelle isole moltissime persone furono schiacciate" 23 .

Ecco, la descrizione geronimiana del miracolo compiuto da sant'Ilarione:

"A quella vista, gli abitanti di Epidauro - constatando con terrore che flutti minacciosi, una massa di onde e montagne di gorghi si abbattevano sui litorali (come, d'altra parte, nelle loro menti li immaginavano già avvenuti) e temendo che la città venisse rovesciata dalle fondamenta - entrarono nel rifugio del vecchio e, quasi partissero per una battaglia, lo depositarono sul litorale. Egli, tracciati tre segni di croce sulla sabbia, stese le mani contro il mare. È incredibile descrivere a quale altezza esso si sia dilatato e fermato davanti a lui e come, fremendo a lungo e quasi accanendosi contro un argine, a poco a poco, sia poi rifluito in se stesso" 24 .

Il segno della croce del quale si serve sant'Ilarione per domare il mare, specialmente nella letteratura monastica, è arma potentissima contro ogni nociva forza demoniaca. I santi, e specialmente i monaci, tracciando il segno della croce, riuscivano sempre a sconfiggere il demonio ${ }^{25}$. Lo stesso san Girolamo così insegna alla vergine Demetriade:

"Mi basti aver colto di volata, dal prato meraviglioso della Scrittura, alcuni fiorellini per esortarti a tener chiusa la stanza del tuo cuore e a munire frequentemente la tua fronte del segno della croce, in modo che lo sterminatore dell'Egitto non trovi appiglio dentro di te, e che i primogeniti, che presso gli Egiziani sono destinati a morire, siano al salvo nella tua anima"26.

${ }^{23}$ Idem, Chronicorum Eusebii Caesariensis continuatio a. 366 c, ed. B. Degórski, in: Hieronymi Opera 15, 288-289: "Terrae motu per totum orbem facto, mare litus egreditur et Siciliae multarumque insularum urbes innumerabiles populos oppressere".

${ }^{24}$ Idem, Vita S. Hilarionis 29, 2-3, Hieronymi Opera 15, 170-173: "Quod cum viderent Epidauritani, frementes scilicet fluctus et undarum moles et montes gurgitum litoribus inferri, verentes, quod iam evenisse cernebant, ne oppidum funditus subverteretur, ingressi sunt ad senem, et quasi ad proelium proficiscentes posuerunt eum in litore. Qui cum tria crucis signa pinxisset in sabulo manusque contra tenderet, incredibile dictu est, in quantam altitudinem intumescens mare ante eum steterit, ac diu fremens et quasi ad obicem indignans paulatim in semetipsum relapsum est".

${ }^{25}$ Cf., ad esempio, Tertullianus Carthaginensis, Adversus Marcionem III 22, 7, 9; Athanasius Alexandrinus, Vita S. Antonii 23, 35. A proposito del segno della croce nell'antichità cristiana, cf. F.J. Dölger, Beiträge zur Geschichte des Kreuzzeichens, JbAC 1 (1958) 5-19; 4 (1961) 5-17; 5 (1962) 5-22; 6 (1963) 7-34; 7 (1964) 5-38; 8-9 (1965-1966) 7-52; 10 (1967) 7-29; G.Q. Reijner, The Terminology of the Holy Cross in Early Christian Literature, Nijmegen 1965; E. Dinkler, Signum crucis, Tübingen 1967; B. Leoni, La croce e il suo segno. Venerazione del segno e culto della reliquia nella antichità cristiana, Verona 1968; V. Grossi, Croce, Crocifisso, DPAC I 864-867 (anche in: NDPAC I 1295-1298).

${ }^{26}$ Hieronymus, Epistula 130, 9, PL 22, 1115: "Haec cursim quasi de prato pulcherrimo sanctarum Scripturarum, parvos flores carpisse sufficiat pro commonitione tui; ut et claudas cubiculum pectoris, et crebro signaculo crucis munias frontem tuam, ne exterminator Aegypti in te locum 
Il Dalmata mette il rilievo l'importanza del segno della croce anche nei due seguenti passi della Vita S. Hilarionis:

"Egli vi ravvisò gli inganni dei demoni e, gettatosi in ginocchio, si tracciò sulla fronte il segno della croce di Cristo e, armato di tale elmo e cinto della corazza della fede, sdraiatosi combatteva con più forza, guardando attorno con occhi inquieti, desiderando vedere in qualche modo coloro udire i quali gli dava terrore" ${ }^{\prime 2}$.

"Dopo esservi giunto, tracciando il segno della croce sul lettuccio di ciascuno e sulle loro membra brucianti di febbre, invocò Gesù. Ed ecco, straordinario prodigio, subito sgorgò contemporaneamente, come da tre fonti, il sudore! Alla stessa ora presero del cibo e, riconoscendo la madre in lacrime e benedicendo Dio, baciarono le mani del santo" 28 .

"Epidauro e tutta quella regione fino a oggi divulgano questo fatto e le madri lo insegnano ai loro figli, affinché ne trasmettano il ricordo ai posteri. Davvero, ciò che fu detto agli Apostoli: «Se crederete, direte a questo monte: 'Passa nel mare', e ciò accadrà ${ }^{29}$, può compiersi anche alla lettera, se uno, però, ha la fede degli Apostoli, tale quale il Signore ordinò loro di avere. Infatti, che importa se un monte che discende nel mare, o se immense montagne di onde si sono d'un tratto irrigidite e, pietrificate solo davanti ai piedi del vecchio, sono rifluite delicatamente dall'altra parte?"30.

Il muro d'acqua che s'arresta dinanzi ad Ilarione e rifluisce resta certamente una delle immagini più efficaci del racconto. Il ricordo del miracolo del Mar Rosso all'Esodo (cf. Es 14, 21-22) è accennato nel gesto del monaco di stendere le mani verso il mare, come fece Mosè. Quanto ai testimoni, è la parola evangelica della fede che sposta le montagne (cf. Mt 17, 20; Mc 11, 23)

reperiat, sed primogenita, quae apud Aegyptios pereunt, in tua mente salventur", trad. italiana di S. Cola, in: San Girolamo, Le Lettere, Introduzione, traduzione, note e indici di Silvano Cola, IV, Roma 1997, 343-344)

${ }^{27}$ Idem, Vita S. Hilarionis 3, 8, Hieronymi Opera 15, 124-125: "Intellexit daemonum ludibria et provolutus genibus crucem Christi signavit in fronte, talique armatus casside et lorica fidei circumdatus iacens fortius proeliabatur, quodammodo videre desiderans, quos horrebat videre et sollicitis oculis huc illucque circumspiciens".

${ }^{28}$ Ibidem 8, 8, Hieronymi Opera 15, 132-133: "Quo postquam venit, singulorum lectulos et ardentia membra consignans, invocavit Iesum. Et, o mira virtus, statim quasi de tribus fontibus sudor pariter erupit; eadem hora acceperunt cibos, lugentemque matrem cognoscentes et benedicentes Deum sancti manus deosculati sunt".

${ }^{29}$ Cf. idem, Commentarii in Evangelium Matthaei III 17, 20.

${ }^{30}$ Idem, Vita S. Hilarionis 29, 4-6, Hieronymi Opera 15, 172-173: "Hoc Epidaurus et omnis illa regio usque hodie praedicat, matresque docent liberos suos ad memoriam in posteros transmittendam. Vere illud, quod ad apostolos dictum est: «Si credideritis, dicetis huic monti: 'Transi in mare', et fiet», etiam iuxta litteram impleri potest, si tamen quis habuerit apostolorum fidem, et talem, qualem illis habendam Dominus imperavit. Quid enim interest, utrum mons descendat in mare, an immensi undarum montes repente obriguerint et, ante senis tantum pedes saxei, ex alia parte molliter fluxerint?". 
a restare impressionata in un insegnamento vivo che sembra accordare stranamente natura sconvolta e miracolo nel limite ad essa imposto.

Ma è anche indubbio che si mescoli all'osservazione oggettiva della potenza delle onde d'un maremoto, la credenza diffusa che, nelle pericolose acque marine, avessero loro privilegiata dimora i demoni, i quali implorano lo stesso monaco: "Ilarione, servo di Dio, perché, a causa tua, non possiamo star tranquilli neanche in mezzo al mare?"31.

La creazione si conferma, pertanto, come teatro naturale sia della divina potenza e bontà, sia dell'insidia di satana, secondo l'insegnamento della Scrittura: "Dio non ha creato la morte e non gode per la rovina dei viventi. Egli infatti ha creato tutto per l'esistenza; le creature del mondo sono sane, in esse non c'è veleno di morte, né gli inferi regnano sulla terra [...] ma la morte è entrata nel mondo per invidia del diavolo" (Sap 1, 13-14; 2, 24).

Non è, dunque, per gusto del fiabesco che la narrazione talvolta si distacchi dall'oggettività dei dati concreti, offrendo ermeneutiche soprannaturali, ma "se il monaco può dominare le infermità e i terrori di questo mondo è perché partecipa profondamente della grazia di Dio che lo colloca in un altro mondo, quello redento, quello sottomesso a Cristo, quello protetto dal Suo amore salvifico, quello escatologico. Se egli non prova timore per i fenomeni anche terribili è perché è unito a Dio e sa che Egli non riserva che bene per colui che ha salvato; se suscita la fede di quanti accorrono a lui è perché la sua persona non è mai schermo a Dio, ma al contrario rivela la bellezza della vita in Lui. La causa del miracolo è la presenza di Dio nel monaco e il suo effetto è una crescita della presenza di Dio fra gli uomini" ${ }^{\prime 2}$.

In cerca di siti sempre più isolati e impervi, sant'Ilarione, lasciata la Dalmazia, raggiunse Cipro, ove morì. Il suo corpo, dapprima sepolto nell'orticello del santo, secondo il suo volere, venne in seguito trafugato dall'amico Esichio e portato in Palestina.

"Tutta la città si meravigliava e la notizia della grandezza del miracolo si era diffusa anche a Salona ${ }^{33}$. Il vecchio, non appena se ne accorse, fuggì nottetempo di nascosto su una barchetta e, incontrata dopo due giorni una nave da carico, si diresse verso Cipro" ${ }^{\prime 4}$.

${ }^{31}$ Ibidem 25, 2, Hieronymi Opera 15, 166-167: "Hilarion, serve Dei, cur nobis per te et in pelago tutos esse non licet?".

${ }^{32}$ B. Degórski, Le tematiche teologiche delle tre Vitae geronimiane, in: The Spirituality of Ancient Monasticism. Acts of the International Colloquium held in Cracow-Tyniec 16-19th November 1994. Specialized Contributions, ed. M. Starowieyski, Pontificia Academia Theologica Cracoviensis, Facultas Theologica, Studia IV/1, Tyniec - Cracow 1995, 190.

${ }^{33}$ Oggi distrutta. Centro amministrativo della provincia della Dalmazia. Situata sulla costa adriatica, a nord dell'odierna Spalato (Split).

${ }^{34}$ Hieronymus, Vita S. Hilarionis 29, 7, Hieronymi Opera 15, 172-173: "Mirabatur omnis civitas et magnitudo signi Salonis quoque percrebuerat. Quod intelligens senex in brevi lembo clam nocte fugit, et inventa post biduum oneraria navi perrexit Cyprum". 
Con queste parole, lo storico Sozomeno così brevemente riassunse ciò che abbiamo presentato sopra:

"Nel periodo qui descritto, anche il monaco Ilarione, ricercato dagli abitanti di Gaza, scappò in Sicilia. Proprio là, raccogliendo la legna nei deserti delle montagne e portandola sulle spalle, vendeva questa merce in città. In tal modo, procurava per sé tale razione giornaliera di cibo che gli bastava per sopravvivere. Quando, però, venne in luce ciò che egli è e che cosa rappresenta con se stesso (infatti, fu tradito da un uomo, appartenente alla categoria dei privilegiati e tormentato dal demonio), Ilarione si recò in Dalmazia, liberando prima quel disgraziato dalla possessione. Dopo aver fatto anche lì, con l'assistenza della forza di Dio, grandi e meravigliosi miracoli, quali l'arresto del mare, attraverso la preghiera, che a causa di un terremoto uscì dai confini inondando la secca, di nuovo si ritirò di là. Infatti, non si intonava con il suo carattere che egli dovesse abitare tra la gente che lo loda. Al contrario, cambiando i posti del suo soggiorno, si sforzava di rimanere in ombra e di soffocare, attraverso questi continui spostamenti, la fama che veniva di lui divulgata. Infine, navigando vicino al Cipro, approdò a Pafos"35.

Il filo conduttore di questi brani, da noi analizzati, della Vita S. Hilarionis è l'operazione dei miracoli, che procura al santo una grande popolarità, sebbene indesiderata e rifuggita dallo stesso Ilarione, il quale mette in atto persino dei sotterfugi per sottrarsene, tra i quali proprio questo che riguarda la Dalmazia, ossia la regione più lontana dalla sua patria. Questa è la caratteristica della Vita: la fuga dagli onori non solo mondani, bensì pure religiosi. Nell'incuranza, nell'insofferenza, quasi nel disprezzo di tali onori bisogna ravvisare, come sempre, una nota autobiografica: è san Girolamo che, essendo ormai un personaggio pubblico, molto più illustre di quanto non fosse al tempo della stesura della Vita S. Pauli Primi Eremitae (stilata negli anni 375-377), sente il disagio della notorietà che contrasta la sua incoercibile aspirazione eremitica: "Ricordava con incredibile desiderio il suo antico modo di vita" 36 , afferma Girolamo di sant'Ilarione, ma sono parole sgorgate dal cuore, cariche di una densa nostalgia, dotate di una tonalità personale.

Il popolino, i vescovi, le matrone romane, i dignitari che accorrono da sant'Ilarione sono gli stessi che ora attorniano Girolamo e l'atteggiamento schivo che ebbe Ilarione viene ora assunto da Girolamo. Anch'egli deve lottare per non lasciarsi lusingare dalla gloria personale e continuare a ispirarsi ai

${ }^{35}$ Sozomenus, HE V 10, 2, PG 67, 1241, trad. italiana di B. Degórski.

${ }^{36}$ Hieronymus, Vita S. Hilarionis 19, 1, Hieronymi Opera 15, 154-155: "flebat quotidie et incredibili desiderio conversationis antiquae recordabatur". 
grandi maestri che avevano saputo ignorarla: "Da nulla più io sono stupito, se non dal modo in cui seppe calpestare la gloria e gli onori" ${ }^{37}$.

Non deve sfuggire questa chiave interpretativa della Vita per non rischiare di considerarla semplicemente una spettacolare e inverosimile carrellata di singoli prodigi; così come non deve sfuggire l'ordine dei miracoli, per non perdere il crescendo che li regge.

I miracoli che avvengono nella parte finale della Vita S. Hilarionis sono descritti più brevemente e sono di più ampio raggio: quasi sempre si tratta di benefici per un'intera città, per tutti i componenti di una nave, per alcuni villaggi sul litorale di un mare in tempesta, e così via. Si nota, inoltre, un'accentuazione del carattere evangelico dei miracoli: i primi riprendono semplicemente le situazioni dei racconti neotestamentari, gli ultimi, invece, tendono a essere la stessa realizzazione delle parole di Cristo. Basti pensare, come abbiamo visto, alla montagna di acqua fermata dal santo, che viene commentata da Girolamo con le parole del Cristo: "Se credete, direte a questo monte: «Passa nel mare», e ciò accadrà" 38 . Si guardi anche all'ultimo miracolo di Ilarione, la guarigione del paralitico, in cui le parole di Ilarione si rifanno a quelle di Cristo: "Alzati e cammina"39.

È stato osservato che, soprattutto nella prima parte, vi è un progressivo aumento di teatralità narrativa, per la quale alcuni episodi risultano esageratamente enfatizzati e costruiti con una tecnica letteraria ricercata e, in certa misura, artificiosa ${ }^{40}$.

È vero, ma questa crescente enfasi è voluta non per mero esercizio letterario, ancor meno per gusto del fiabesco. Essa è voluta da san Girolamo, raffinato scrittore e sensibile autore monastico, per esprimere l'ascesa del santo davanti a Dio e davanti agli uomini. Bisogna tenere presente il fatto che l'operare miracoli, nel contesto monastico, rispecchia anzitutto l'unione del monaco con Dio. Se egli può dominare le infermità e i terrori di questo mondo, è perché partecipa profondamente della grazia di Dio che lo colloca in un altro mondo, quello redento, quello sottomesso a Cristo, quello protetto dal suo amore salvifico, quello escatologico. Se egli non prova timore dei fenomeni anche terribili, è perché è unito a Dio e sa che Dio non riserva che bene per colui che ha salvato; se suscita la fede in quanti accorrono a lui, è perché la sua persona non è mai schermo a Dio, ma al contrario rivela la bellezza della vita in lui.

La causa dei miracoli è la presenza di Dio nel monaco e il suo effetto è una crescita della presenza di Dio fra gli uomini.

${ }^{37}$ Ibidem 20, 1, Hieronymi Opera 15, 156-157: "ego nihil ita stupeo, quam gloriam illum et honorem calcare potuisse".

${ }^{38}$ Ibidem 29, 4, Hieronymi Opera 15, 172-173: "Si credideritis, dicetis huic monti: «Transi in mare», et fiet (cf. Mt 17, 20; Mc 11, 23)".

${ }^{39}$ Ibidem 31, 7, Hieronymi Opera 15, 176-177: "Surge et ambula (cf. Mt 9, 5; At 3, 6)".

${ }^{40}$ Cf. Ch. Mohrmann, Introduzione, in: Vita di Martino [Sulpicio Severo]. Vita di Ilarione [Girolamo]. In memoria di Paola [Girolamo], introd. di Ch. Mohrmann, testo critico e commento a cura di A.A.R. Bastiaensen - J.W. Smit, traduzioni di L. Canali - C. Moreschini, Vite dei Santi IV, Milano 1975, XLVI-XLVII. 
Di nuovo, dunque, come per la Vita S. Pauli Primi Eremitae di san Girolamo, protagonista è la presenza di Dio e a Dio: "recitava le Scritture come fosse presente Dio" "41, scrive di sant'Ilarione, quasi fugacemente, Girolamo all'inizio della Vita, e tutto ciò che segue ne sarà la manifestazione.

Con l'aumentare della popolarità del santo, cresce anche il suo desiderio di solitudine, inversamente proporzionale alla gloria esteriore. Per esprimere ciò, ecco che san Girolamo ricorre a fughe sempre più rocambolesche; dal semplice spostamento, a mano a mano Ilarione escogita modi e luoghi più ardui.

Al di là, dunque, della congerie di dettagli che si incontrano nella Vita $S$. Hilarionis e della ricchezza di notizie che si confanno alla passione storica e geografica di Girolamo ${ }^{42}$, 1'architettura geronimiana è lineare: la vita eremitica si espande nella compassione per il prossimo, che viene portato alla fede dalla testimonianza del monaco. L'ascesi eremitica, quindi, non è finalizzata solo alla santificazione del monaco, ma alla testimonianza esteriore dell'amore di Dio e alla sua glorificazione nell'universo. In questo senso, la Vita S. Hilarionis può essere considerata la continuazione della precedente, quella della Vita S. Pauli Primi Eremitae, e le note autobiografiche di san Girolamo una fase successiva della sua maturazione di monaco.

\section{THE SOJOURN OF ST. HILARION OF GAZA IN DALMATIA ACCORDING TO THE VITA S. HILARIONIS OF ST. JEROME}

\section{(Summary)}

The narrative of the sojourn of St. Hilarion in Dalmatia within the Vita written by St. Jerome seems to encompass and synthetize all the principal elements of the biography of this Palestinian hermit. The search for solitude and anonymity amidst the populations of far distant lands, the impossibility of hiding - for the sake of charity - God's operating power in miracles, the very presence of the Lord in the faithful monk living a life of prayer and penance, humbleness and love for God, the capability of showing the Lord's presence in deeds, and the autobiographical references of the author of the Vita, St. Jerome, to himself through the life of Hilarion are all elements - though present in the brief section concerning the events occurred in Dalmatia - which profile the author.

It is of significance his resorting to Biblical references, especially to the Gospel, highlighting the relevance of faith. St. Hilarion carries out the work of Christ and presents himself as "alter Christus". The victory over the sea monster is the victory over evil-the devil and over death. The dominion over nature's power, namely over the power of the waters of the seismic wave, endangering the earth and its inhabitants. It is - through Christ, with Christ and in Christ, Word of the Living

${ }^{41}$ Hieronymus, Vita S. Hilarionis 4, 3, Hieronymi Opera 15, 126-127: "Scripturas quoque sanctas memoriter tenens post orationes et psalmos quasi Deo praesente recitabat".

${ }^{42} \mathrm{Cf}$. Mohrmann, Introduzione, p. XLVII-XLVIII. 
God - the victory of life over the primordial chaos, as well as the new creation over the wound of the original sin. St. Hilarion proposes himself as a "living gospel", proclaiming and revealing the mystery of the Kingdom amidst humankind. For this reason his lamp, namely he himself as the shining glory of the light of Christ, cannot remain under the bowl, although he would have liked so but, on the contrary, it is God's will that the lamp be put on its stands, so that everyone may see it, be enlightened by the truth and glorify the Heavenly Father (Mt 5:14-16).

Finally, the passages selected and analyzed - though brief in content - witness of the wide classical knowledge of the Dalmatian, who does quote Latin works and authors containing references to events and people of the past. Especially in those pages whom St. Jerome devotes to "his land", Dalmatia, he seems to grant the highest homage to his Christian hero.

\author{
POBYT ŚW. HILARIONA Z GAZY W DALMACJI \\ WEDŁUG VITA S. HILARIONIS ŚW. HIERONIMA
}

\title{
(Streszczenie)
}

Opis pobytu mnicha Hilariona w Dalmacji, przekazany przez św. Hieronima w Vita S. Hilarionis, zdaje się zawierać i streszczać wszystkie główne przesłania i treści, które znajdują się w tej biografii świętego mnicha palestyńskiego: poszukiwanie samotności i bycia nieznanym pośród ludów w dalekich stronach, niemożność ukrycia się w imię miłości, Boża moc działająca poprzez wielkie cuda, która świadczy o tym, że w wiernym i rozmodlonym, pokutującym mnichu, pełnym pokory względem Boga i bliźniego jest obecny Pan, objawiający swoją chwałę za jego pośrednictwem.

Nie bez znaczenia jest fakt, iż Hieronim nawiązuje tu wyraźnie do znanych fragmentów Ewangelii, które podkreślają znaczenie wiary mnicha. Święty kontynuuje dzieło Chrystusa na ziemi i jawi się jako "alter Christus". Zwycięstwo odniesione nad morskim potworem jest zwycięstwem nad złem, diabłem i śmiercią. Panowanie nad siłami natury, w naszym przypadku: nad żywiołem rozhuczanego morza, które grozi zniszczeniem ziemi i ludzi, staje się przez Chrystusa, z Chrystusem i w Chrystusie zwycięstwem życia nad pierwotnym chaosem i grzechem pierworodnym. Hilarion jawi się więc jako żyjąca „Ewangelia”, która głosi i jednocześnie objawia tajemnicę Królestwa istniejącego już wśród ludzi.

W tych krótkich fragmentach ujawnia się także wielka erudycja Hieronima oparta na znajomości kultury klasycznej. Wydaje się, że właśnie na tych stronicach poświęconych ojczyźnie - Dalmacji - Hieronim złożył największy hołd temu palestyńskiemu mnichowi.

Key words: St. Jerome, St. Hilarion of Gaza, Dalmatia, Hagiography, Monasticism.

Parole chiave: san Girolamo, sant' Ilarione di Gaza, Dalmazia, agiografia, monachesimo. 
Słowa kluczowe: św. Hieronim, św. Hilarion z Gazy, Dalmacja, hagiografia, monastycyzm.

\section{BIBLIOGRAFIA}

\section{Fonti}

Ammianus Marcellinus, Res gestae, ed. W. Seyfarth: Ammiani Marcellini Rerum gestarum libri qui supersunt, I-II, Leipzig 1978.

Athanasius Alexandrinus, Vita S. Antonii, ed. G.J.M. Bartelink, SCh 400, Paris 1994.

Gaius Plinius Secundus, Naturalis historia, in: http://penelope.uchicago.edu/Thayer/L/ Roman/Texts/Pliny_the_Elder/8*.html (consultato il 9 I 2017).

Hieronymus, Epistulae, PL 22, 325-1224, trad. italiana di S. Cola, in: San Girolamo, Le Lettere, Introduzione, traduzione, note e indici di Silvano Cola, I-IV, Roma 1996-1997.

Hieronymus, Chronicorum Eusebii Caesariensis continuatio, ed. B. Degórski, in: Hieronymi, Historica et hagiographica. Vita Beati Pauli monachi Thebaei. Vita Hilarionis. Vita Malchi monachi captivi. Epistula praefatoria in Chronicis Eusebii Caesariensis. Chronicorum Eusebii Caesariensis continuatio. De viris inlustribus. In Regulae S. Pachomii versionem praefatio $=$ Girolamo, Opere storiche e agiografiche. Vita di san Paolo, eremita di Tebe. Vita di Ilarione. Vita di Malco, l'eremita prigioniero. Prefazione alla traduzione delle Cronache di Eusebio di Cesarea. Continuazione delle Cronache di Eusebio di Cesarea. Gli uomini illustri. Prefazione alla traduzione della Regola di Pacomio, Hieronymi Opera 15 = Opere di Girolamo 15, Roma 2014, 236-307.

Hieronymus, Commentarii in Evangelium Matthaei, ed. D. Hurs - M. Adriaen, CCL 77, Turnhout 1969.

Hieronymus, Explanationes in Esaiam, ed. R. Maisano: Hieronymi, Explanationes in Esaiam. Girolamo = Commento a Isaia, Hieronymi Opera 4/1-4, Roma 2013.

Hieronymus, Vita S. Hilarionis, ed. B. Degórski, in: Hieronymi, Historica et hagiographica. Vita Beati Pauli monachi Thebaei. Vita Hilarionis. Vita Malchi monachi captivi. Epistula praefatoria in Chronicis Eusebii Caesariensis. Chronicorum Eusebii Caesariensis continuatio. De viris inlustribus. In Regulae S. Pachomii versionem praefatio = Girolamo, Opere storiche e agiografiche. Vita di san Paolo, eremita di Tebe. Vita di Ilarione. Vita di Malco, l'eremita prigioniero. Prefazione alla traduzione delle Cronache di Eusebio di Cesarea. Continuazione delle Cronache di Eusebio di Cesarea. Gli uomini illustri. Prefazione alla traduzione della Regola di Pacomio, Hieronymi Opera 15 = Opere di Girolamo 15, Roma 2014, 116-181.

Hieronymus, Vita S. Pauli Primi Eremitae, ed. B. Degórski, in: Hieronymi, Historica et hagiographica. Vita Beati Pauli monachi Thebaei. Vita Hilarionis. Vita Malchi monachi captivi. Epistula praefatoria in Chronicis Eusebii Caesariensis. Chronicorum Eusebii Caesariensis continuatio. De viris inlustribus. In Regulae S. Pachomii versionem praefatio = Girolamo, Opere storiche e agiografiche. Vita di san Paolo, eremita di Tebe. Vita di Ilarione. Vita di Malco, l'eremita prigioniero. Prefazione alla traduzione delle Cronache di Eusebio di Cesarea. Continuazione delle Cronache di Eusebio di Cesarea. Gli uomini illustri. Prefazione alla traduzione della Regola di Pacomio, Hieronymi Opera 15 = Opere di Girolamo 15, Roma 2014, 72-115.

Marcus Tullius Cicero, Philippicae, in: https://la.wikisource.org/wiki/In_M._Antonium Oratio_-_Philippica_II\#.5B29.5D (consultato il 9 I 2017).

Paulus Orosius, Historia adversus paganos, ed. C. Zangemeister, CSEL 5, Vindobonae 1982, 1-600. 
Publius Terentius Afer, Hecyra, in: http://latin.packhum.org/loc/134/5/4\#4 (consultato il 9 I 2017).

Socrates, Historia ecclesiastica, PG 67, 29-872.

Sozomenus, Historia ecclesiastica, PG 67, 843-1630.

Tertullianus Carthaginensis, Adversus Marcionem, ed. A. Kroymann, CCL 1, Turnhout $1954,437-726$.

\section{Studi}

Boblitz H., Die Allegorese der Arche Noah in der frühen Bibelauslegung, "Frühmittelalterliche Studien" 6 (1972) 159-170.

DANiéLou J., Déluge, baptême, jugement, “Dieu vivant” 8 (1947) 97-112.

DANiÉLou J., Sacramentum futuri. Études sur les origines de la typologie biblique, Paris 1950, 55-94 (Noé et le déluge).

DegóRsKi B., Le tematiche teologiche delle tre Vitae geronimiane, in: The Spirituality of Ancient Monasticism. Acts of the International Colloquium held in Cracow-Tyniec 16$19^{\text {th }}$ November 1994. Specialized Contributions, ed. M. Starowieyski, Pontificia Academia Theologica Cracoviensis, Facultas Theologica, Studia IV/1, Tyniec - Cracow 1995, 183-196.

DegóRski B., La natura del deserto nelle "Vitae" di san Girolamo, in: La cultura scientifico-naturalistica nei Padri della Chiesa (I-V sec.). XXXV Incontro di studiosi dell'antichità cristiana, Roma, 4-6 maggio 2006, Istituto Patristico "Augustinianum", SEA 101, Roma 2007, 549-578.

Degórski B., Przyroda pustyni jako ,stworzenie” u św. Hieronima, in: Wiedza w stużbie dobra człowieka, ed. M. Włosiński, Włocławek 2010, 71-82.

DinkLer E., Signum crucis, Tübingen 1967.

DöLGER F.J., Beiträge zur Geschichte des Kreuzzeichens, JbAC 1 (1958) 5-19; 4 (1961) 5-17; 5 (1962) 5-22; 6 (1963) 7-34; 7 (1964) 5-38; 8-9 (1965-1966) 7-52; 10 (1967) 7-29.

Féghali P., La figura di Noè secondo i SS. Padri (Contributo alla storia della esegesi), "Studii biblici Franciscani liber annuus" 20 (1970) 138-165.

FÉghali P., Note sur l'exégèse de Saint Éphrem. Commentaire sur le déluge (Gn 6, 19-9. 17), ParOr 8 (1977-1978) 67-86.

Gribomont J., Ilarione di Gaza, DPAC II 1753 (anche in: NDPAC II 2528).

LEONI B., La croce e il suo segno. Venerazione del segno e culto della reliquia nella antichità cristiana, Verona 1968.

Mohrmann Ch., Introduzione, in: Vita di Martino [Sulpicio Severo]. Vita di Ilarione [Girolamo]. In memoria di Paola [Girolamo], introd. di Ch. Mohrmann, testo critico e commento a cura di A.A.R. Bastiansen - J.W. Smit, traduzioni di L.Canali - C. Moreschini, Vite dei Santi IV, Milano 1975, IX-LXI.

Opelt I., Des Hieronymus Heiligenbiographien als Quellen der historischen Topographie des östlichen Mittelmeerraumes, RQ 74 (1979) 145-177.

Praga G., La leggenda di S. Ilarione a Epidauro, "Archivio Storico della Dalmazia” 25 (1938) 81-91.

ReIJNER G.Q., The Terminology of the Holy Cross in Early Christian Literature, Nijmegen 1965.

Grossi V., Croce, Crocifisso, DPAC I 864-867 (anche in: NDPAC I 1295-1298).

Wilmart A., Arca Noë, RBen 26 (1909) 1-12. 